\title{
A numerical and experimental study of sand-rubber mixtures subjected to oedometric compression
}

\author{
Pravin Badarayani ${ }^{1, *}$, Patrick Richard ${ }^{1}$, Bogdan Cazacliu ${ }^{1}$, Riccardo Artoni ${ }^{1}$, and Erdin Ibraim $^{2}$ \\ ${ }^{1}$ IFSTTAR,MAST/GPEM, Bouguenais, 44340 Nantes, France \\ ${ }^{2}$ University of Bristol, Faculty of Engineering, Queens Building, Bristol BS8 1 TR, UK
}

\begin{abstract}
The stockpiling of waste tires at landfill sites has become a nuisance for the society. One of the alternatives could be converting the recycled rubber into powdered form and mixing it with soil to use it as the backfill of the retaining structures. This paper is based on the study of such sand-rubber mixtures. In this work, Discrete Element (DEM) simulations were employed to study the mechanical response of sand-rubber mixtures with respect to a column of grains enclosed within a rigid cylindrical confinement, and subjected to an oedometric compression by the fixed velocity displacement of one of the horizontal walls. Further, experimental analysis was also carried out by using a uniaxial load cell to load the sand-rubber mixtures under compression. Different initial packings of sand-rubber mixture were prepared by varying:

(a) the packing volume fraction and

(b) the volume fraction of rubber.

The mechanical response at small strains was studied for these sand-rubber packings. The mixture behavior was observed to be more sand-dominant or rubber-dominant depending on the rubber fraction and the mixture quality. Moreover, variation in the initial volume fraction of the packing also caused a difference in the load bearing of the packings for a given strain and a given rubber fraction.
\end{abstract}

\section{Introduction}

The volume of scrap tires is increasing at an alarming rate throughout the world. It is a frequent sight that the used tires are stockpiled on the landfill sites. It is posing a hindrance to the development of the society from an environmental point of view and hence it is of utmost importance to tackle this problem on a global scale. The researchers as well as the industries throughout the world are continuously working towards finding a sustainable solution for their disposal. The use of such waste tires has become quite common in some thermochemical technologies of energy generation such as tire pyrolysis, incineration, etc. However, inspite of its use in the energy generation, the amount of waste tires still does not seem to have been much affected. This has caused an increasing interest in the usage of recycled rubber (in powder or shredded form) from waste tires in combination with other materials such as cement, soil, asphalt,etc. for geotechnical applications [1-3]. An advantage of using rubber as an additive is its properties viz. lightweight, elasticity, good damping capacity, etc. which makes it an interesting choice for such applications. In this respect, a lot of work has been done and is in progress to test the viability of mixing the recycled rubber chips with soil. The sand-rubber mixture can be an interesting solution for geotechnical applications such as the backfill of the retaining structures,

*e-mail: pravin.badarayani@ifsttar.fr highway embankments and reinforced soil structures [47]. The dynamic properties of these mixtures allow their use in a damping system e.g. machine foundations or railroad track beds as well to reduce the vibrations since rubber has a high damping capacity [8]. The sand-rubber mixtures also have excellent drainage capabilities and are hence useful in filtration applications [9].

There have been quite extensive numerical as well as experimental studies to analyze the behavior of sandrubber mixtures within the past few years. The deformation characteristics of sand-rubber mixtures have been studied by the means of triaxial tests with an aim to use the mixtures as backfill in the retaining structures [10]. There have been several works to study the behavior of sandrubber mixtures by the use of Discrete Element Method (DEM) [11-14]. They extensively quantify the effect of different factors such as sand-rubber size ratio, rubber fraction, etc. on the mechanical behavior of sand-rubber mixtures. A typical model has been provided for the isotropic compression loading of the sand-rubber mixtures in order to present an idea that rubber particles fill the voids of the system thus reducing the void ratio with the increasing pressure[15].

This paper accounts for the numerical DEM and experimental tests which were conducted on sand-rubber mixtures under oedometer loading conditions. The mechanical behavior of these packings has been quantified to em- 
phasize the effect of the packing volume fraction and the rubber fraction on the sand-rubber mixture.

\section{Method: Numerical study}

\subsection{Assembly of initial packings using DEM simulations}

The first step in the Discrete Element simulations was the preparation of initial packing. It consisted of 9000 sand grains. The assembly process was initialized by defining important parameters viz. coefficient of friction, cylinder size (the vessel defined for the deposition of the grains), Young's modulus of elasticity, grain size, etc. These important parameters are given in Table 1. For the sake of simplicity, the grains were considered to be monodisperse and spherically shaped, thus neglecting the shape and size effects. Subsequently, the Velocity Verlet Algorithm was implemented to calculate the positions and velocities of each grain at every time step and the Newton's equations of motion were solved. The Discrete Element Method considers the grains to be non-deformable. However, a small overlap between the contacting sand and rubber grains was considered which in turn facilitated the calculation of the contact forces as shown in Fig.1. Here, the Hertz model was used to calculate the normal forces while the tangential forces were calculated using the Coulomb friction model. Hence,

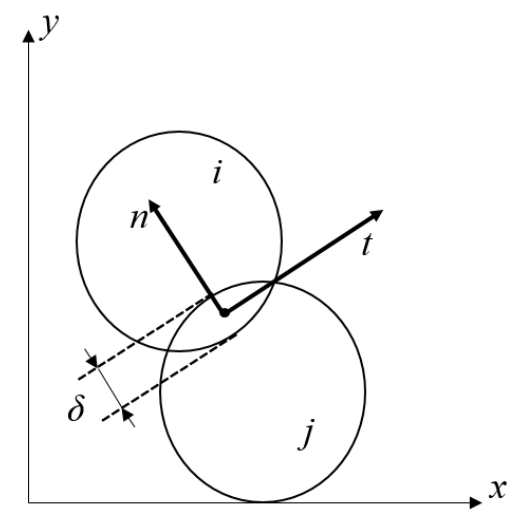

Fig. 1. Figure showing the interaction between two contacting grains, $i$ and $j$ wherein $\delta$ is the overlap between the two grains, $n$ and $t$ are the normal and tangential vectors of the contact forces respectively

$$
F_{n, \text { elas }}=K_{n} \delta^{3 / 2} \text {, }
$$

where, $F_{n, \text { elas }}$ is the normal elastic contact force, $K_{n}$ is the stiffness and $\delta$ is an exaggerated overlap between the contacting grains. The actual overlap is much smaller than the overlap shown in Fig. 1. Hence, it has been signified as an exaggerated overlap. The normal stiffness is given by:

$$
K_{n}=\frac{4}{3} E_{e f f} \sqrt{R_{e f f}},
$$

where, $E_{\text {eff }}$ is the effective Young's modulus of elasticity for the contacting $i$ and $j$ particle given by, $E_{\text {eff }}=$ $E_{i} E_{j} / E_{i}+E_{j}$ and similarly $R_{e f f}$ is the effective radius of the contacting grains $i$ and $j$ given by, $R_{\text {eff }}=$ $R_{i} R_{j} / R_{i}+R_{j}$. To account for dissipation, a viscous-like force is chosen to ensure a velocity-independent normal coefficient of restitution, $e_{n}[16]$ :

$$
F_{\text {diss }, n}=\frac{-\sqrt{5} \ln e_{n}}{\ln ^{2} e_{n}+\pi^{2}} \sqrt{m_{e f f} K_{n}} \delta^{1 / 4} \dot{\delta} .
$$

The tangential force is given by,

$$
F_{t}=K_{t} \Delta
$$

where $K_{t}$ is the tangential stiffness and $\Delta$ is the incremental tangential displacement. Since it is based on the Coulomb's law, the criterion for sliding betwen the grains is $F_{t}>\mu_{t} F_{n}$. Consequently the tangential displacement $\Delta$ is truncated to satisfy the latter inequality. In addition, a limiting condition for average grain deformation was imposed in order to ensure that the DEM is valid and also a small deformation of rubber is considered (rubber being more deformable than sand). The simulations were stopped once the average grain deformation (for rubberrubber type of contact) due to the compression exceeded the order of $10^{-2}$.

After defining all the necessary parameters mentioned before, the process started with the deposition of the grains within the cylinder of pre-defined dimensions. Due to the presence of the gravity, the grains started settling at the bottom of the cylinder. Consequently, the cylinder started filling up with the new grains occupying the positions above the previously stabilised grains in the cylinder. The process continued till the packing had achieved a state of equilibrium. This packing thus obtained was 100\% sand grain packing.

\subsection{Sand-rubber mixture preparation by varying packing volume fraction and rubber fraction}

After the preparation of the initial packings by the deposition of sand grains under gravity, the sand rubber mixtures were prepared by defining the properties of some sand grains with that of the rubber grains. Consequently, different sand-rubber mixtures were prepared as a function of the rubber fraction (by volume). Moreover, the initial packing volume fraction was also varied by varying the intergranular and grain-wall friction coefficients. The respective packing volume fractions are 0.62 which will be denoted as dense packing and 0.57 which will be called as loose packing. The values of the friction coefficients used were 0.1 for dense packings and 0.5 for loose packings. Since, the replacement of sand grains by rubber grains changes the equlibrium of the packing due to the difference in the sand and rubber properties, the packings were allowed to relax and stabilize before the actual compression was imposed.

\subsection{Discrete Element Method: Oedometric Compression}

The sand rubber packings prepared were simulated using the Discrete Element Method (DEM) with a threedimensional, velocity imposed oedometric compression 
Table 1. Table showing the different parameters to define the initial packing

\begin{tabular}{rc}
\hline \hline Parameter & Value \\
\hline $\begin{array}{r}\text { Number of grains } \\
\text { Young's modulus of elasticity (sand) }\end{array}$ & 9000 \\
Young's modulus of elasticity (rubber) & $700 \mathrm{MPa}$ \\
Mean radius of grains & $1 \mathrm{MPa}$ \\
Coefficient of friction between the grains $(\mu)$ & $0.25 \mathrm{~mm}$ \\
Coefficient of friction between the grains and wall $\left(\mu_{W}\right)$ & 0.1 (dense packing), 0.5 (loose packing) \\
Cylinder radius (Rcyl) & $4.95 \mathrm{~mm}$ \\
Cylinder height & $12.98 \mathrm{~mm}$ (dense packing), $13.69 \mathrm{~mm}$ (loose packing) \\
\hline
\end{tabular}

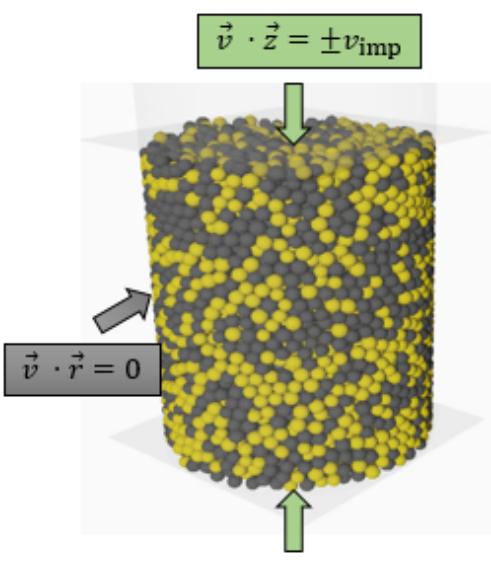

Fig. 2. Figure showing the setup for the DEM simulations: $v_{i} m p$ is the imposed compression velocity in the direction of $\mathrm{Z}$ axis and the packing is laterally constrained

model (see Fig.2). As mentioned before, the initial packings were allowed to relax before the actual compression. The relaxation time was fixed by verifying whether the mean position of the packing height was stable for a sufficient time period. The process began by defining the initial positions and velocities of the grains. The grains in the top-most and the bottom-most layers of the packing were given fixed opposite velocities in order to compress the packing. The advantage of DEM is that it allows to capture detailed informations of the interparticle contacts. Thus, it helped to study the mechanical behavior of the sandrubber mixtures due to one-dimensional compression.

\section{Method: Experimental Study}

\subsection{Laboratory fabrication of sand-rubber mixtures}

The sand and rubber used for the laboratory scale experiments were Leighton Buzzard sand and recycled tire rubber respectively. Both sand and rubber used were in the size fraction range of $1.6 \mathrm{~mm}-2 \mathrm{~mm}$. Moist tamping method was used for the sand-rubber mixture fabrication. It is one of the widely used methods for the preparation of sand-rubber mixtures [17]. The process started with the measurement of the sand and rubber in terms of mass (calculated as per volume for the required void ratio) in a mixing bowl. The height of the sand-rubber samples was fixed to be $39.9 \mathrm{~mm}$. Hence, the entire sample was prepared in two equal layers. The measured quantities of sand and rubber were mixed by adding $10 \%$ of water (by volume of sand) in a mixing bowl. They were then thoroughly mixed using a spoon to ensure a good distribution of the rubber particles throughout the mixture. After mixing, the mixture was then deposited in the oedometer cell from a zero drop height in two successive layers as mentioned before. Each layer was compacted using a steel rod till the required sample height and void ratio was achieved. The fabrication process is shown in Fig. 3. During the labo-

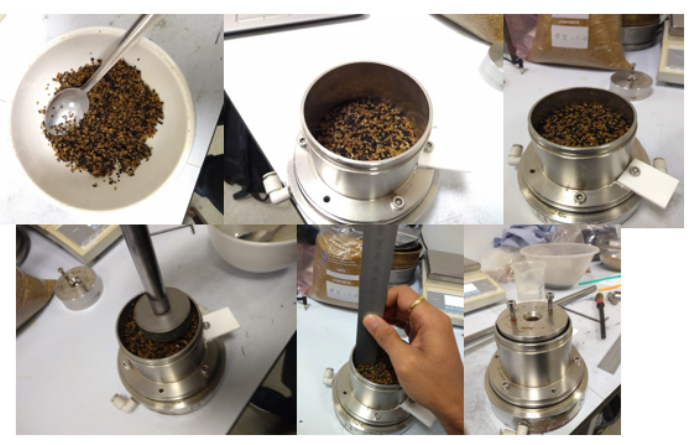

Fig. 3. Figure showing the preparation of sand-rubber mixtures using moist tamping method

ratory preparation as well, the two variations in the sandrubber mixtures were achieved i.e. packing volume fraction and the rubber volume fraction. For different packing volume fractions as well, the same sample fabrication process was adopted. The varying factors were the volumes of sand and rubber.

\subsection{Experimental setup and oedometric compression}

The sand-rubber samples prepared were then tested using a uniaxial load cell apparatus. The test setup consists of a load cell, a Linear Variable Displacement Transducer (LVDT), oedometer cell with the test sample, water tank and acquisition system (see Fig. 4). Since, water has been added during the mixing of sand and rubber, it was necessary to ensure that the the bridges formed between the particles were broken and thus minimize the effect of water addition. Hence, the tests were performed under saturated 


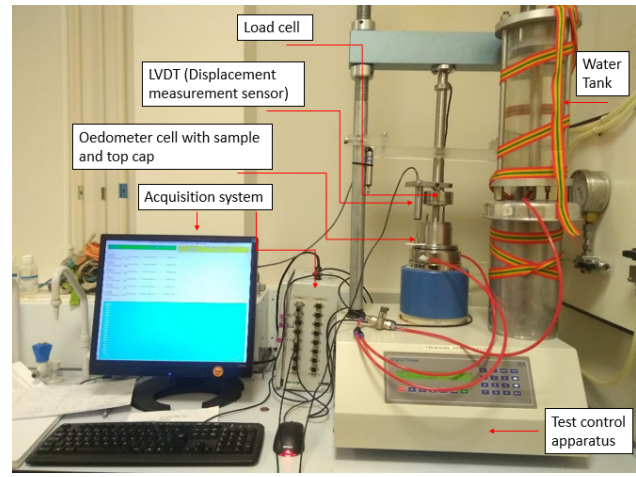

Fig. 4. Figure showing the experimental setup for the oedometric compression tests

condition. For this purpose, water from the water tank was let in through the inlets of the oedometer cell until the sample was completely saturated. The platform was adjusted using the test control apparatus in order to bring the load cell in contact with the top cap of the oedometer cell. Once ensured that the load cell has been properly seated on the top cap, the values for the LVDT and the load cell in the program for the data acqusition were set to zero. Consequently, in the test control appartus, the velocity was set as $0.5 \mathrm{~mm} / \mathrm{min}$ for all the tests. The compression test was stopped once the force reached the load cell limiting value $(10 \mathrm{kN})$. The oedometer cell was then removed, the height with the top cap was measured and then the top cap was removed and the sand-rubber sample was allowed to relax. A final measurement of height was also done after the relaxation.

\section{Stress vs Strain: Influence of packing volume fraction and rubber fraction}

The main purpose behind preparing two initial packings with different packing volume fractions was to study its effect on the mechanical behavior of the sand-rubber packing. Fig. 5 shows the results for the dense and loose packings of all rubber fractions obtained from the numerical simulations using DEM. The results depict the change in the mechanical response of the sand-rubber mixtures with the varying rubber fraction. The stiffness of the packing decreases with the increasing rubber fraction. Moreover, it can be observed in Fig. 5 that for the same value of strain and same rubber fraction, the dense and loose packings have different stress levels. Fig. 6 shows the results for dense and loose packings obtained from the experiments. The packing volume fraction for the dense and loose packings constructed in the experiments was 0.606 and 0.571 respectively. It can be seen that the experimental results follow a similar trend as that of the results from the numerical simulations.

It should however be noted that the value of stress in the numerical simulations is quite low with respect to the real time experiments. The reason for this are the values of Young's modulus of elasticity used for sand and rubber which are quite low in the numerical simulations as
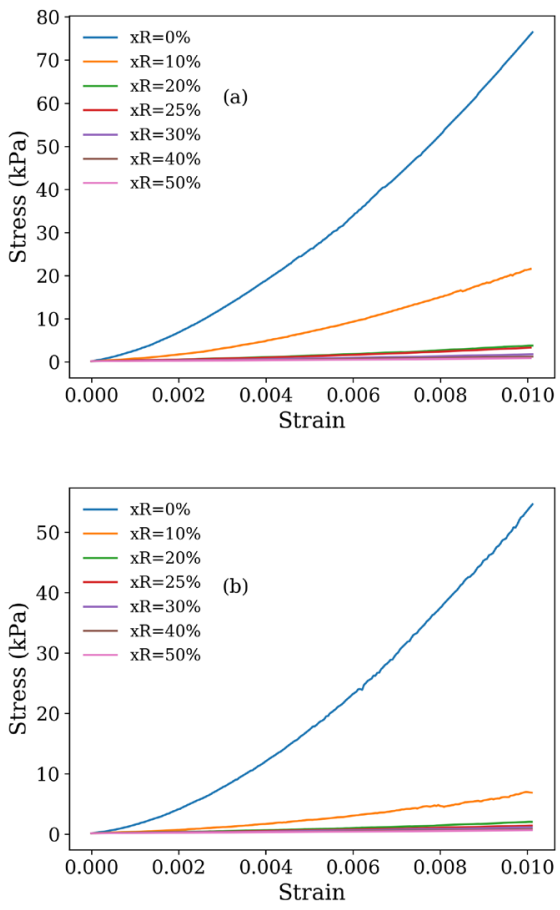

Fig. 5. Stress vs strain for (a)dense packing and (b)loose packing obtained from numerical simulations using DEM
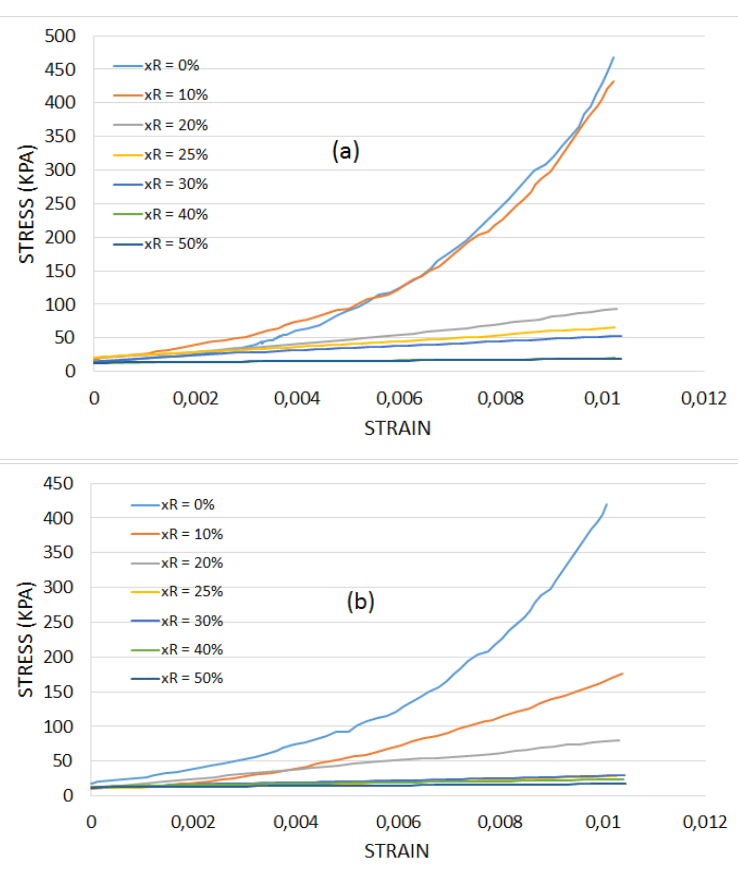

Fig. 6. Stress vs strain for (a)dense packing and (b)loose packing obtained from experiments

compared to the real-time values. In addition, the grains used in the numerical simulations are perfectly spherical which is not possible in the case of experiments. Also, the sand-rubber packings are not built using the same process in the case of numerical simulations and experiments. Further, there is a difference in the level of homogeneity of the sand-rubber mixtures in experiments and numerical 
simulations. Moreover, the imposed compression velocity is different for the experiments and DEM simulations. For all these mentioned reasons, the results should be studied only for a qualitative comparison instead of a direct quantitative comparison.

Fig. 5 and Fig. 6 suggest that the results obtained from the DEM simulations and the experimental results are in good agreement with each other qualitatively.

The mechanical response observed in Fig. 5 and Fig. 6 can be easily understood. The global mechanical response of a granular packing is mainly controlled by the strong force network [18]. With the increasing rubber fraction, the probability to involve rubber particles in this strong force network increases, hence explaining why the stiffness of the mixture decreases with increasing $x_{R}$. It can be observed from the plots that, in experiments, the stiffness of the packing obtained for $x_{R}=10 \%$ is very close to that obtained for $x_{R}=0 \%$. This suggests that only a few rubber grains are involved in the strong force network. But, this is not the case numerically, probably because the packings formed in the numerical simulations are more homogeneous (clusters are more likely to form in experiments than in the case of simulations). The plots shown here clearly justify that for higher volume fraction, the number of rubber grains involved in the strong force networks is significant and thus influences directly the mixture stiffness.

\section{Conclusion and Perspectives}

Oedometric compression tests were performed using both DEM numerical simulations as well as laboratory experiments. The mechanical response of the sand-rubber mixtures was studied. The sand-rubber mixture samples were prepared for two different packing volume fractions and increasing rubber volume fractions. The response of the packings was found to be lying within two ranges viz. sand-dominated and rubber-dominated. With the increasing rubber fraction, for the same volume of solids, the volume of rubber increases and hence the sand force chains decrease in the number. Consequently, it was observed that the stiffness of the sand-rubber mixtures decreased with the increasing rubber fraction. The results obtained from the numerical simulations and the laboratory experiments were found to be in good qualitative agreement with each other.

\section{References}

[1] M. Irfan, Y. Ali, S. Ahmed, I. Hafeez, Arabian Journal for Science and Engineering 43, 1795 (2018)

[2] A.H. Farhan, A.R. Dawson, N.H. Thom, Materials and Design 97, 98 (2016)

[3] M. Elchalakani, T. Aly, E. Abu-Aisheh, Australian Journal of Civil Engineering 14, 1 (2016)

[4] J. Lee, R. Salgado, A. Bernal, C. Lovell, Journal of geotechnical and geoenvironmental engineering 125, 132 (1999)
[5] P.J. Bosscher, T.B. Edil, S. Kuraoka, Journal of Geotechnical and Geoenvironmental Engineering 123, 295 (1997)

[6] V.K. Garga, V. O'shaughnessy, Canadian Geotechnical Journal 37, 75 (2000)

[7] P.S. Poh, B.B. Broms, Journal of performance of constructed facilities 9, 76 (1995)

[8] Z.Y. Feng, K.G. Sutter, Geotechnical Testing Journal 23, 338 (2000)

[9] J.R. Valdes, T.M. Evans, Canadian Geotechnical Journal 45, 588 (2008)

[10] S. Youwai, D.T. Bergado, Canadian Geotechnical Journal 40, 254 (2003)

[11] J.L. Perez, C. Kwok, K. Senetakis, Computers and Geotechnics 80, 199 (2016)

[12] D.R. Eidgahee, E.S. Hosseininia, Mechanical behavior modeling of sand-rubber chips mixtures using discrete element method (DEM), in AIP Conference Proceedings (AIP, 2013), Vol. 1542, pp. 269-272

[13] C. Lee, H. Shin, J.S. Lee, International Journal for Numerical and Analytical Methods in Geomechanics 38, 1651 (2014)

[14] M. Asadi, A. Mahboubi, K. Thoeni, Granular Matter 20, 18 (2018)

[15] A. Platzer, S. Rouhanifar, P. Richard, B. Cazacliu, E. Ibraim, Granular Matter 20, 81 (2018)

[16] D. Antypov, J.A. Elliott, EPL (Europhysics Letters) 94, 50004 (2011)

[17] S. Rouhanifar, Ph.D. thesis, University of Bristol (2017)

[18] F. Radjai, M. Jean, J.J. Moreau, S. Roux, Phys. Rev. Lett. 77, 274 (1996) 